\title{
Anomalous Scaling in the Kinematic Magnetohydrodynamic Turbulence
}

\author{
Eva Jurčišinová ${ }^{1, \star}$, Marián Jurčišin ${ }^{1, \star \star}$, and Richard Remecký ${ }^{1,2, \star \star \star}$ \\ ${ }^{1}$ Institute of Experimental Physics, Slovak Academy of Sciences, \\ Watsonova 47, 04001 Košice, Slovak Republic \\ ${ }^{2}$ Bogoliubov Laboratory of Theoretical Physics, Joint Institute for Nuclear Research, \\ Joliot-Curie 6, 141980 Dubna, Moscow Region, Russian Federation
}

\begin{abstract}
The problem of the anomalous scaling in the kinematic magnetohydrodynamic turbulence is investigated using the field theoretic renormalization group method and the operator product expansion technique. The anomalous dimensions of all leading composite operators, which drive the anomalous scaling of the correlation functions of a weak passive magnetic field, are determined up to the second order of the perturbation theory (i.e., in the two-loop approximation in the field theoretic terminology) in the presence of a large scale anisotropy for physically the most interesting three-dimensional case. It is shown that the leading role in the anomalous scaling properties of the model is played by the anomalous dimensions of the composite operators near the isotropic shell, in accordance with the Kolmogorov's local isotropy restoration hypothesis. The importance of the two-loop corrections to the anomalous dimensions of the leading composite operators is demonstrated.
\end{abstract}

\section{Introduction}

The understanding on a fundamental level of the existence of the anomalous scaling in turbulent systems, i.e., the existence of deviations from the form of the inertial-range scaling predicted by the classical Kolmogorov-Obukhov theory [1,2], belongs among the most intriguing open problems in the theory of fully developed turbulence. Although the problem of the anomalous scaling of various correlation functions of the genuine turbulent velocity field is still open, during the last three decades, a significant progress has been made in the investigation of the anomalous scaling properties of the correlation functions of various passive scalar or vector fields advected by the Gaussian as well as nonGaussian turbulent velocity fields (see, e.g., Refs. [3-8] and references cited therein). In this respect, in Refs. [6, 7], the so-called Kazantsev-Kraichnan model with given Gaussian statistics of the turbulent velocity field $[9,10]$ was investigated using the field theoretic renormalization group (RG) approach [11] in the two-loop approximation. As was shown in Refs. [6, 7], the anomalous inertial-range scaling behavior of the single-time two-point correlation functions of the weak magnetic field is much more pronounced than the anomalous behavior of the corresponding structure functions of the passive scalar

\footnotetext{
^e-mail: jurcisine@saske.sk

$\star \star$ e-mail: jurcisin@saske.sk

$\star \star \star$ e-mail: remecky@saske.sk
} 
in the Kraichnan model with the same velocity field statistics [12]. Here, the importance of the twoloop calculations is naturally dictated by the fact that on the one-loop level of the corresponding perturbation expansion the both models exhibit the same scaling behavior. Note that the same is also valid in the corresponding models of passively advected scalar and vector (magnetic) fields by the non-Gaussian turbulent velocity field driven by the stochastic Navier-Stokes equation [4, 13]. Thus, at least two-loop field theoretic renormalization group analysis is again necessary here. However, while the two-loop results for the scalar case are already known for a long time [4], the corresponding two-loop analysis of the problem of passive advection of the weak magnetic field in the framework of the kinematic magnetohydrodynamic (MHD) turbulence is still absent. In the present paper, we begin the systematic investigation of the scaling properties of the magnetic field in the kinematic MHD turbulence using the field theoretic renormalization group technique in the two-loop approximation. As we shall see, the anomalous scaling in the magnetic case is significantly much more pronounced than in the scalar case [4].

\section{Anomalous scaling in the kinematic MHD turbulence}

The kinematic MHD model of a passively advected weak solenoidal magnetic field $\mathbf{b} \equiv \mathbf{b}(x)[x \equiv$ $(t, \mathbf{x})]$ is described by the following system of stochastic equations

$$
\begin{aligned}
\partial_{t} \mathbf{b} & =v_{0} u_{0} \Delta \mathbf{b}-(\mathbf{v} \cdot \partial) \mathbf{b}+(\mathbf{b} \cdot \partial) \mathbf{v}+\mathbf{f}^{\mathbf{b}} \\
\partial_{t} \mathbf{v} & =v_{0} \Delta \mathbf{v}-(\mathbf{v} \cdot \partial) \mathbf{v}-\partial P+\mathbf{f}^{\mathbf{v}},
\end{aligned}
$$

where $\mathbf{v} \equiv \mathbf{v}(x)$ is the incompressible turbulent velocity field, $\partial_{t} \equiv \partial / \partial t, \partial_{i} \equiv \partial / \partial x_{i}, \Delta \equiv \partial^{2}$ is the Laplace operator, $v_{0}$ is the viscosity coefficient (subscript 0 denotes bare parameters of the unrenormalized theory), $v_{0} u_{0}$ is the bare coefficient of magnetic diffusivity ( $u_{0}$ is reciprocal magnetic Prandtl number), $P \equiv P(x)$ is the pressure, and $\mathbf{f}^{\mathbf{b}}=\mathbf{f}^{\mathbf{b}}(x)$ and $\mathbf{f}^{\mathbf{v}}=\mathbf{f}^{\mathbf{v}}(x)$ represent random noises.

The Gaussian random noise $\mathbf{f}^{\mathbf{b}}=\mathbf{f}^{\mathbf{b}}(x)$ is taken with the correlator in the general form $D_{i j}^{b}\left(x ; x^{\prime}\right) \equiv$ $\left\langle f_{i}^{b}(x) f_{j}^{b}\left(x^{\prime}\right)\right\rangle=\delta\left(t-t^{\prime}\right) C_{i j}\left(\left|\mathbf{x}-\mathbf{x}^{\prime}\right| / L\right)$ through which the large scale anisotropy can be introduced into the system (see, e.g., Ref. [13] for details) but its explicit form is unimportant in what follows. On the other hand, the statistics of the random noise $\mathbf{f}^{\mathbf{v}}=\mathbf{f}^{\mathbf{v}}(x)$ is also Gaussian with zero mean and the following explicit form of the pair correlation function

$$
D_{i j}^{v}\left(x ; x^{\prime}\right) \equiv\left\langle f_{i}^{v}(x) f_{j}^{v}\left(x^{\prime}\right)\right\rangle=\delta\left(t-t^{\prime}\right) \int \frac{d^{d} \mathbf{k}}{(2 \pi)^{d}} D_{0} k^{4-d-2 \varepsilon} P_{i j}(\mathbf{k}) e^{i \mathbf{k} \cdot\left(\mathbf{x}-\mathbf{x}^{\prime}\right)},
$$

where $P_{i j}(\mathbf{k})=\delta_{i j}-k_{i} k_{j} / k^{2}$ is the ordinary transverse projector, $d$ denotes the spatial dimension of the system, $D_{0} \equiv g_{0} v_{0}^{3}>0$ is the positive amplitude, $g_{0}$ plays the role of the coupling constant of the model, and the physical value of the formally small parameter $0<\varepsilon \leq 2$ is $\varepsilon=2$.

Using the well-known theorem [14] the stochastic problem (1)-(3) can be transformed into the corresponding field theoretic model of the double set of fields $\Phi=\left\{\mathbf{v}, \mathbf{b}, \mathbf{v}^{\prime}, \mathbf{b}^{\prime}\right\}$ with the following action functional

$$
\begin{aligned}
S(\Phi) & =\frac{1}{2} \int d t_{1} d^{d} \mathbf{x}_{\mathbf{1}} d t_{2} d^{d} \mathbf{x}_{\mathbf{2}}\left[v_{i}^{\prime}\left(x_{1}\right) D_{i j}^{v}\left(x_{1} ; x_{2}\right) v_{j}^{\prime}\left(x_{2}\right)+b_{i}^{\prime}\left(x_{1}\right) D_{i j}^{b}\left(x_{1} ; x_{2}\right) b_{j}^{\prime}\left(x_{2}\right)\right] \\
& +\int d t d^{d} \mathbf{x}\left\{\mathbf{v}^{\prime}\left[-\partial_{t}+v_{0} \Delta-(\mathbf{v} \cdot \partial)\right] \mathbf{v}+\mathbf{b}^{\prime}\left[-\partial_{t} \mathbf{b}+v_{0} u_{0} \Delta \mathbf{b}-(\mathbf{v} \cdot \partial) \mathbf{b}+(\mathbf{b} \cdot \partial) \mathbf{v}\right]\right\},
\end{aligned}
$$

where $x_{i}=\left(t_{i}, \mathbf{x}_{\mathbf{i}}\right), i=1,2, \mathbf{v}^{\prime}$ and $\mathbf{b}^{\prime}$ are added auxiliary transverse fields which have the same tensor properties as the fields $\mathbf{v}(x)$ and $\mathbf{b}(x)$, and required summations over dummy indices are assumed. 
The model described by the action (4) is multiplicatively renormalizable and the scaling behavior of various correlation functions of the model in the inertial range is driven by the existence of the infrared stable fixed point of the renormalization group equations (see Refs. [13, 15] for details). The issues of interest are especially various equal-time two-point quantities. Such quantities are the equaltime two-point correlation functions

$$
B_{N-m, m}(r) \equiv\left\langle b_{r}^{N-m}(t, \mathbf{x}) b_{r}^{m}\left(t, \mathbf{x}^{\prime}\right)\right\rangle, \quad r=\left|\mathbf{x}-\mathbf{x}^{\prime}\right|,
$$

where $b_{r}$ denotes the component of the magnetic field directed along the vector $\mathbf{r}=\mathbf{x}-\mathbf{x}^{\prime}$. They exhibit the following scaling behavior for $r / l \gg 1$ and fixed $r / L$ (here, $L$ represents the energy pumping scale of the model and $l$ represents the dissipation scale with the inertial range defined by $l \ll r \ll L$ )

$$
B_{N-m, m}(r) \simeq v_{0}^{-N / 2}(r / l)^{N(1-\varepsilon / 3)-\gamma_{N-m}^{*}-\gamma_{m}^{*}} R_{N, m}(r / L),
$$

where $\gamma_{N-m}^{*}$ and $\gamma_{m}^{*}$ are the anomalous dimensions of the composite operators $b_{r}^{N-m}$ and $b_{r}^{m}$ taken at the fixed point and $R_{N, m}(r / L)$ are the so-called scaling functions. The asymptotic behavior of the scaling functions $R_{N, m}(r / L)$ deep inside the inertial interval, i.e., in the limit $r / L \rightarrow 0$, can be performed by using the OPE technique [16]) with the following representation

$$
R_{N, m}(r / L)=\sum_{i} C_{F_{i}}(r / L)(r / L)^{\Delta_{F_{i}}}, \quad r / L \rightarrow 0 .
$$

Here, the summation is performed over all possible renormalized composite operators $F_{i}$ allowed by the symmetry of the problem with critical dimensions $\Delta_{F_{i}}$ and the corresponding coefficient functions $C_{F_{i}}(r / L)$ are regular in $r / L$. Note that the very existence of the nontrivial anomalous scaling in the model is related to the existence of the so-called "dangerous" composite operators with negative critical dimensions. In our case, with the large scale anisotropy given by the unit vector $\mathbf{n}$, the central role is played by the operators constructed solely from the magnetic field $\mathbf{b}(x)$ in the following form

$$
F_{N, p}=[\mathbf{n} \cdot \mathbf{b}]^{p}(\mathbf{b} \cdot \mathbf{b})^{l}, \quad N=2 l+p .
$$

Then the final inertial range scaling behavior of the correlation functions (5) has the form

$$
B_{N-m, m}(r) \simeq v_{0}^{-N / 2}(r / l)^{-\gamma_{N-m}^{*}-\gamma_{m}^{*}}(r / L)^{\gamma_{N}^{*}} \sim r^{-\gamma_{N-m}^{*}-\gamma_{m}^{*}+\gamma_{N}^{*}},
$$

where $\gamma_{M}^{*}$ are the corresponding anomalous dimensions of the leading composite operators (8) near the isotropy shell $\left(\gamma_{M}^{*}=\gamma_{M, 0}^{*}\right.$ for even values of $M$ and $\gamma_{M}^{*}=\gamma_{M, 1}^{*}$ for odd values of $M$ ).

In the two-loop approximation, the anomalous dimensions $\gamma_{N, p}^{*}$ are determined by the analysis of the corresponding set of one- and two-loop composite operators graphical representation of which is the same as in the scalar model [4]. In this respect, one can write

$$
\gamma_{N, p}^{*}=\gamma_{N, p}^{*(1)} \varepsilon+\gamma_{N, p}^{*(2)} \varepsilon^{2}+O\left(\varepsilon^{3}\right),
$$

where $\gamma_{N, p}^{*(1)}$ represents the one-loop contribution and $\gamma_{N, p}^{*(2)}$ is the corresponding two-loop correction to the anomalous dimension $\gamma_{N, p}^{*}$. The explicit form of the one-loop correction $\gamma_{N, p}^{*(1)}$ can be found, e.g., in Ref. [4]. On the other hand, the explicit expression for the two-loop correction $\gamma_{N, p}^{*(2)}$ calculated in the present paper is very large and will be given elsewhere.

Our analysis shows that the two-loop corrections are much more important in the present vector case than in the corresponding model of advection of the passive scalar field [4]. For example, in Fig. 1 the behavior of the total two-loop anomalous dimension $\gamma_{3,1}^{*}$ is compared to the corresponding one-loop result $\gamma_{3,1}^{*(1)} \varepsilon$ as a function of $\varepsilon$ for $d=3$. 


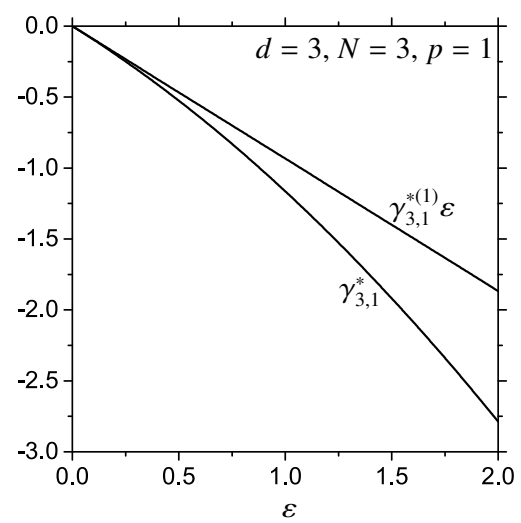

Figure 1. Comparison of the two-loop anomalous dimension $\gamma_{3,1}^{*}$ to the one-loop result $\gamma_{3,1}^{*(1)} \varepsilon$ for $d=3$.

\section{Conclusion}

Using the field theoretic RG method and the operator product expansion technique, we have investigated the importance of two-loop corrections to the anomalous dimensions of the leading composite operators that drive the anomalous scaling of the correlation functions of the magnetic field in the kinematic MHD turbulence. It is shown that two-loop corrections play a much more important role in the present vector model than in the case of the analogous model of a passive scalar advection [4].

\section{Acknowledgement}

The work was supported by grants VEGA: 2/0065/17 and No. 2/0058/19 and by APVV-17-0020.

\section{References}

[1] A.S. Monin, A.M Yaglom, Statistical Fluid Mechanics (MIT Press, Cambridge, MA, 1975)

[2] U. Frisch, Turbulence: The Legacy of A. N. Kolmogorov (Cambridge University Press, Cambridge, 1995)

[3] G. Falkovich, K. Gawedzki, M. Vergassola, Rev. Mod. Phys. 73, 913 (2001)

[4] L.Ts. Adzhemyan, N.V. Antonov, J. Honkonen, T. L. Kim, Phys. Rev. E 71, 016303 (2005)

[5] N.V. Antonov, J. Phys. A: Math. Gen. 39, 7825 (2006)

[6] N.V. Antonov, N.M. Gulitskiy, Phys. Rev. E 85, 065301 (2012)

[7] E. Jurčišinová, M. Jurčišin, J. Phys. A: Math. Theor. 45, 485501 (2012)

[8] E. Jurčišinová, M. Jurčišin, M. Menkyna, Phys. Rev. E 95, 053210 (2017)

[9] R.H. Kraichnan, Phys. Fluids 11, 945 (1968)

[10] A.P. Kazantsev, Sov. Phys. JETP 26, 1031 (1968)

[11] L.Ts. Adzhemyan, N.V. Antonov, A.N. Vasil'ev, The Field Theoretic Renormalization Group in Fully Developed Turbulence (Gordon and Breach, London, 1999)

[12] L.Ts. Adzhemyan, N.V. Antonov, A.N. Vasil'ev, Phys. Rev. E 58, 1823 (1998)

[13] E. Jurčišinová, M. Jurčišin, R. Remecký, J. Phys. A: Math. Theor. 42, 275501 (2009)

[14] A.N. Vasil'ev, Quantum-Field Renormalization Group in the Theory of Critical Phenomena and Stochastic Dynamics (Chapman \& Hall/CRC, Boca Raton, 2004)

[15] E. Jurčišinová, M. Jurčišin, R. Remecký, Phys. Rev. E 84, 046311 (2011)

[16] J. Zinn-Justin, Quantum Field Theory and Critical Phenomena (Clarendon, Oxford, 1989) 